\title{
NEUTRON STARS IN THE RELATIVISTIC HARTREE-FOCK THEORY AND HADRON-QUARK PHASE TRANSITION
}

\author{
B. Y. SUN* \\ School of Physics, Peking University, \\ Beijing, 100871, China \\ *E-mail: sunbaoyuan@gmail.com \\ U. LOMBARDO and G. F. BURGIO \\ Istituto Nazionale di Fisica Nucleare, Laboratori Nazionali del Sud and Sezione di Catania, Via \\ Santa Sofia 64, 95123, Italy

\section{J. MENG} \\ School of Physics, Peking University, \\ Beijing, 100871, China \\ Institute of Theoretical Physics, Chinese Academy of Sciences, \\ Beijing, 100080, China \\ Center of Theoretical Nuclear Physics, National Laboratory of Heavy Ion Accelerator, \\ Lanzhou, 730000, China \\ E-mail: mengj@pku.edu.cn
}

\begin{abstract}
Based on the density-dependent relativistic Hartree-Fock theory (DDRHF) for hadronic matter, the properties of neutron stars have been studied and compared with the results from the density-dependent relativistic mean field theory (DDRMF). Though similar equations of state are obtained, DDRHF calculations give larger fractions of proton, electron and muon at high baryon density for neutron star matter than the ones from DDRMF. The maximum masses of neutron stars lie between $2.3 \mathrm{M}_{\odot}$ and $2.5 \mathrm{M}_{\odot}$, and the corresponding radii between $11.7 \mathrm{~km}$ and $12.5 \mathrm{~km}$. In addition, the phase transition from hadronic matter to quark matter in neutron stars is studied by using the MIT bag model for the quark phase. The transition is studied in both Gibbs and Maxwell constructions.
\end{abstract}

Keywords: Many-body theory; Relativistic models; Nuclear matter; Neutron stars; Bag model; Phase transitions.

\section{Introduction}

Neutron stars provide a natural laboratory for exploring the baryonic matter at high densities, well exceeding in the center the nuclear saturation density of $\rho_{\text {sat }}=$ $0.16 \mathrm{fm}^{-3}$. Recently, new results from the observations of neutron star properties have been reported which provide stringent constraints on the equation of state (EoS) of strongly interacting matter at high densities, see Ref. 1,2 and references therein. 
On the description of nuclear matter and finite nuclei, within the relativistic scheme, the mean field theory has achieved great success during the past years. One of the most outstanding models is the relativistic Hartree approach with the no-sea approximation, namely the relativistic mean field (RMF) theory. ${ }^{3-5}$ In recent years, RMF models with density dependent nucleon-meson couplings (DDRMF) have been developed.$^{6-9}$ However, in the framework of the RMF approach, the Fock terms are dropped out, which may have remarkable effects on nuclear matter especially at high density.

During the past decades, there were several attempts to include the Fock term in the relativistic descriptions of nuclear systems. ${ }^{10-13}$ Recently, a new RHF method, so called, density-dependent relativistic Hartree-Fock (DDRHF) theory ${ }^{14-16}$ has brought us a new in-sight into this problem. With the effective Lagrangians of Refs. 14,15 , the DDRHF theory can quantitatively describe the ground state properties of many nuclear systems on the same level as RMF.

The appearance of quark matter in the interior of massive neutron stars is one of the main issues of astrophysics. Many EoS have been used to describe the interior of neutron stars. ${ }^{17-19}$ Due to the impact of recent experiments in heavy-ion collisions $^{20}$ and new observational limits for the mass and the mass-radius relationship of compact stars, ${ }^{21-24}$ affected by large theoretical uncertainties for quark matter, the question whether a pure quark phase exists in the interior of neutron stars or not still have not yet received a clear answer.

The paper is organized as follows: In Sec. 2 we review the nuclear EoS within the relativistic Hartree-Fock theory and the quark matter EoS within the MIT bag model. In Sec. 3 we present the results for the neutron star structure in DDRHF and the hadron to quark phase transition. Section 4 contains our conclusions.

\section{Theoretical Framework}

\subsection{Hadronic Phase: The Relativistic Hartree-Fock Theory}

In the present work, we study nuclear matter properties based on the new developed density dependent relativistic Hartree-Fock theory. The details of the DDRHF theory can be found in Ref. 14-16. The DDRHF theory starts from an effective Lagrangian density where nucleons are described as Dirac spinors interacting via exchange of several mesons $(\sigma, \omega, \pi$ and $\rho)$ and the photons. Using the Legendre transformation and the equations of motion for the mesons and photon field operators, the Hamiltonian can be written in a form which includes only nucleon degree of freedom,

$$
\begin{aligned}
H & =\int d^{3} x[\bar{\psi}[-i \boldsymbol{\gamma} \cdot \boldsymbol{\nabla}+M] \psi] \\
& +\frac{1}{2} \int d^{3} x d^{4} y \sum_{i=\sigma, \omega, \rho, \pi, A} \bar{\psi}(x) \bar{\psi}(y) \Gamma_{i}(x, y) D_{i}(x, y) \psi(y) \psi(x),
\end{aligned}
$$


where $\Gamma_{i}(x, y)$ is the interaction vertex of the respective mesons, and $D_{i}(x, y)$ is the corresponding meson propagator.

Generally, we can expand the nucleon field operator $\psi$ into a complete set of Dirac spinors $u(p, s, \tau)$

$$
\psi(x)=\sum_{p, s, \tau} u(p, s, \tau) e^{-i p x} c_{p, s, \tau},
$$

where $c_{p, s, \tau}$ denote annihilation operators for nucleons in the state $(p, s, \tau)$. Notice that the no-sea approximation is assumed here. On such a basis, we can construct the trial ground state $\left|\Phi_{0}\right\rangle=\prod_{i=1}^{A} c_{p, s, \tau}^{\dagger}|0\rangle$, where $|0\rangle$ is the physical vacuum. From the above trial state, we can build up the energy functional by taking the expectation value of Hamiltonian (1):

$$
E \equiv\left\langle\Phi_{0}|H| \Phi_{0}\right\rangle=E_{k}+\left(E_{\sigma}^{D}+E_{\omega}^{D}+E_{\rho}^{D}\right)+\left(E_{\sigma}^{E}+E_{\omega}^{E}+E_{\rho}^{E}+E_{\pi}\right),
$$

wherein the exchange terms are given by

$$
E_{i}^{E}=-\frac{1}{2} \sum_{p_{1}, s_{1}, \tau_{1}} \sum_{p_{2}, s_{2}, \tau_{2}} \bar{u}\left(p_{1}, s_{1}, \tau_{1}\right) \bar{u}\left(p_{2}, s_{2}, \tau_{2}\right) \frac{\Gamma_{i}(1,2)}{m_{i}^{2}+\boldsymbol{q}^{2}} u\left(p_{1}, s_{1}, \tau_{1}\right) u\left(p_{2}, s_{2}, \tau_{2}\right) .
$$

Then, the self-energy can be determined by the self-consistent variation of the energy functional, namely

$$
\Sigma(p) u(p, s, \tau)=\frac{\delta}{\delta \bar{u}(p, s, \tau)} \sum_{\sigma, \omega, \rho, \pi}\left[E_{i}^{D}+E_{i}^{E}\right] .
$$

Generally, it can also be written as

$$
\Sigma\left(p, p_{F}\right)=\Sigma_{S}\left(p, p_{F}\right)+\gamma_{0} \Sigma_{0}\left(p, p_{F}\right)+\gamma \cdot \hat{\boldsymbol{p}} \Sigma_{V}\left(p, p_{F}\right),
$$

where $\hat{\boldsymbol{p}}$ is the unitary vector along $\boldsymbol{p}$, and $p_{F}$ is the Fermi momentum. Here, the tensor term $\gamma_{0} \boldsymbol{\gamma} \cdot \hat{\boldsymbol{p}} \Sigma_{T}\left(p, p_{F}\right)$ is omitted because it does not appear in the HartreeFock approximation for the nuclear matter.

In this work, density-dependent meson-nucleon couplings will be used as introduced in Ref. 6 . For the coupling constant $g_{\pi}$, the exponential density dependence is adopted as $g_{\pi}\left(\rho_{v}\right)=g_{\pi}(0) e^{-a_{\pi} x}$. Three new DDRHF parameter sets PKO1, $\mathrm{PKO} 2, \mathrm{PKO} 3^{14-16}$ have been used in recent calculations.

The chemical potential can be calculated from self-energies

$$
\mu=E_{F}=\Sigma_{0}\left(p_{F}\right)+\sqrt{\left[p_{F}+\Sigma_{V}\left(p_{F}\right)\right]^{2}+\left[M+\Sigma_{S}\left(p_{F}\right)\right]^{2}} .
$$

In cold neutron star matter, the chemical potentials must fulfill the condition equilibrium under weak interaction, i.e., $\mu_{p}=\mu_{n}-\mu_{e}$ and $\mu_{\mu}=\mu_{e}$. Moreover, the baryon number conservation, $\rho_{b}=\rho_{n}+\rho_{p}$, as well as the condition of charge neutrality, $\rho_{p}=\rho_{n}+\rho_{e}$, must be satisfied. Then the pressure can be obtained from the thermodynamic relation

$$
P\left(\rho_{v}\right)=\rho_{v}^{2} \frac{d}{d \rho_{v}} \frac{E}{\rho_{v}}=\sum_{i=n, p, e, \mu} \rho_{i} \mu_{i}-E .
$$




\subsection{Quark Phase: The MIT Bag Model}

We now turn to briefly describe the bulk properties of uniform quark matter, deconfined from the $\beta$-stable hadronic matter as mentioned in the previous section. Here we use the MIT bag model. ${ }^{25}$ The thermodynamic potential of $q=u, d, s$ quarks can be expressed as a sum of the kinetic term and the one-gluon-exchange term, ${ }^{26,27}$

$$
\begin{aligned}
\Omega_{q}\left(\mu_{q}\right)= & -\frac{3 m_{q}^{4}}{8 \pi^{2}}\left[\frac{y_{q} x_{q}}{3}\left(2 x_{q}^{2}-3\right)+\ln \left(x_{q}+y_{q}\right)\right]+\alpha_{s} \frac{3 m_{q}^{4}}{2 \pi^{3}}\left\{\left[y_{q} x_{q}-\ln \left(x_{q}+y_{q}\right)\right]^{2}\right. \\
& \left.-\frac{2}{3} x_{q}^{4}+\ln y_{q}+2 \ln \left(\frac{\sigma_{\text {ren }}}{m_{q} y_{q}}\right)\left[y_{q} x_{q}-\ln \left(x_{q}+y_{q}\right)\right]\right\}
\end{aligned}
$$

where $m_{q}$ and $\mu_{q}$ are the $q$ current quark masses and the chemical potential, respectively, and $y_{q}=\mu_{q} / m_{q}, x_{q}=\sqrt{y_{q}^{2}-1} . \alpha_{s}$ denotes the QCD fine structure constant, whereas $\sigma_{\text {ren }}=313 \mathrm{MeV}$ is the renormalization point. In this work we will consider massless $u$ and $d$ quarks (together with $m_{s}=150 \mathrm{MeV}$ ), and choose $\alpha_{s}=0$ since it has no remarkable influence on neutron star bulk properties. ${ }^{28}$

The number density $\rho_{q}$ of $q$ quarks is related to $\Omega_{q}$ via $\rho_{q}=-\partial \Omega_{q} / \partial \mu_{q}$. Then the total energy density and pressure for the quark system are given by

$$
\epsilon_{\mathrm{Q}}\left(\rho_{u}, \rho_{d}, \rho_{s}\right)=\sum_{q}\left(\Omega_{q}+\mu_{q} \rho_{q}\right)+B, \quad P_{Q}(\rho)=\sum_{q} \mu_{q} \rho_{q}-\epsilon_{Q} .
$$

where $B$ is the energy density difference between the perturbative vacuum and the true vacuum, i.e., the bag constant. In the following we present results based on the MIT model using constant values of the bag constant, $B=90,120,150 \mathrm{MeV} / \mathrm{fm}^{3}$.

The composition of $\beta$-stable quark matter is determined by imposing the condition of equilibrium of the chemical potentials under weak interaction: $\mu_{d}=\mu_{s}=$ $\mu_{u}+\mu_{e}$. As in baryonic matter, the equilibrium relations between the chemical potentials must be supplemented with the charge neutrality condition and the total baryon number conservation in order to determine the chemical composition $\rho_{f}(\rho)$.

\section{Results and Discussion}

\subsection{Neutron Star Properties in The DDRHF Theory}

We now study the neutron star properties in the DDRHF theory. For comparison, we also perform the calculations with four DDRMF interactions: TW99, ${ }^{6}$ DD-ME1, ${ }^{7}$ DD-ME2, ${ }^{8}$ PKDD. ${ }^{9}$ In Fig. 1(a) are shown the symmetry energies as a function of the baryon density for different DDRMF and DDRHF EoSs. At high density, three DDRHF interactions PKO1, PKO2 and PKO3 give sizable enhancement for the symmetry energies than DDRMF ones, while PKDD give different results from other DDRMF EoSs. These remarkable distinctions would have a large influence on the cooling behavior of neutron stars. According to recent analyses, ${ }^{1,29}$ an acceptable EoS shall not allow direct Urca processes to occur in neutron stars with masses below $1.5 \mathrm{M}_{\odot}$, otherwise it will be in disagreement with modern observational soft 
X-ray data in the temperature-age diagram. This constrains the density dependence of the nuclear asymmetry energy which should not be too strong. From Fig. 1 (b), one can see that the DDRHF results cannot be in agreement with this constraint. In particular, for PKO1, D-Urca will occur at fairly low mass $1.2 \mathrm{M}_{\odot}$. However, there are still several uncertainties concerning the cooling mechanism of neutron stars, ${ }^{30}$ and this constraint may not be considered as a stringent one and there could be other mechanisms, e.g. appearance of quark phase, to solve this problem.

The EoSs of $\beta$-stable neutron star matter are shown in Fig. 2(a). Three DDRHF interactions give the similar trend to DD-ME1 and DD-ME2 in DDRMF, while PKDD give a little softer one and TW99 have softest EoS. As a result, the maximum

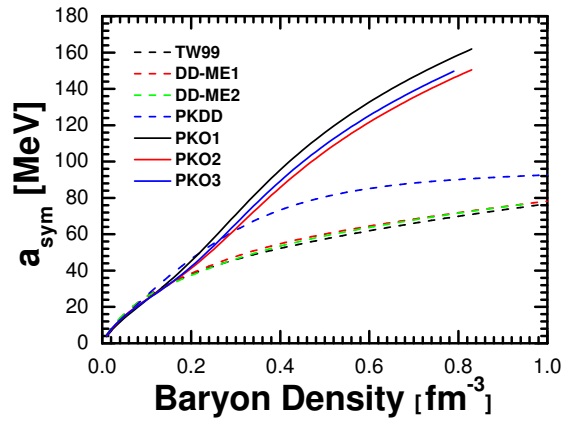

(a)

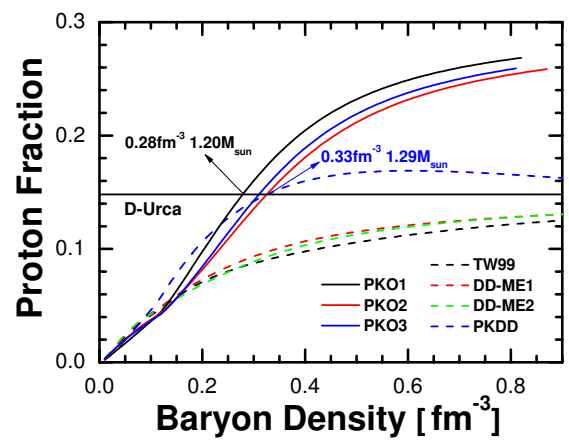

(b)

Fig. 1. (a) The symmetry energies for different EoSs. (b) Proton fractions in neutron star matter for different EoSs. The line labeled with D-Urca is threshold for happening direct Urca process.

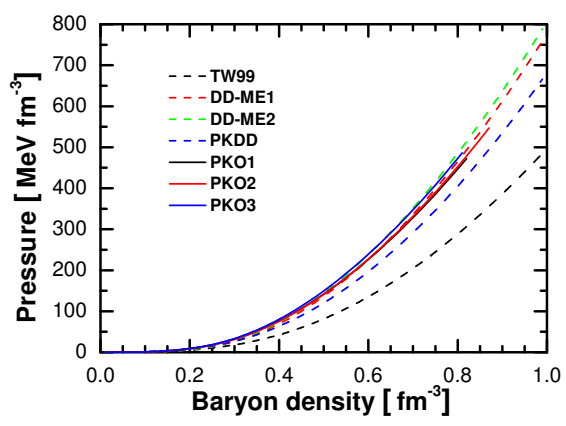

(a)

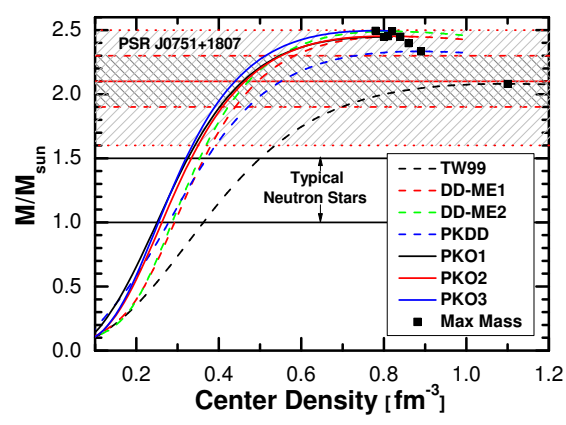

(b)

Fig. 2. (a) Pressure vs. baryon density of the neutron star matter for different nuclear EoSs. (b) Mass vs. central density of compact stars for different nuclear EoSs. Filled squares denote the maximum mass configurations. The observational constraints are taken from Fig. 2 in Ref. 1 
masses of neutron stars lie between $2.3 \mathrm{M}_{\odot}$ and $2.5 \mathrm{M}_{\odot}$ in DDRHF, as shown in Fig. 2 (b), consisting with observational constraint from PSR J0751+1807. ${ }^{21}$ Furthermore, mass-radius relations of pure neutron stars are shown in Fig. 4 (a). One can see DDRHF have a better aggreement with three observational limits ${ }^{22-24}$ than DDRMF, especially for EXO 0748-676. ${ }^{24}$ In DDRMF, TW99 gives smaller maximum mass and radius than others, which is not consistent with constraints.

\subsection{Hadron-Quark Phase Transition in Neutron Star Matter}

We now consider the hadron-quark phase transition in neutron stars. Both the simple Maxwell construction and the more general Gibbs (Glendenning) construction ${ }^{31}$ are adopted to treat the phase transition. In the Maxwell construction, the transition is determined by the intersection points between the hadronic and the quark phase in the plot of pressure versus baryonic (neutron) chemical potential. After projecting this crossing point onto the plot of density versus baryonic (neutron) chemical potential, one can get the corresponding transition densities from lowdensity baryonic matter, $\rho_{H}$, to high-density quark matter, $\rho_{Q}$. So between these two densities, there will be a plateau for pressure in the EoS curve, seen in Fig. 3 (a). This sudden density increase would make stars to be unstable when the gravitational interaction is taken into account. In addition, from the figure we find that, when bag constant of the MIT bag model increases, the pressure of phase transition occurring will also increase, and the transition density will go up, too.

In the Gibbs construction, there are two conserved charges. Hence, a mixed phase

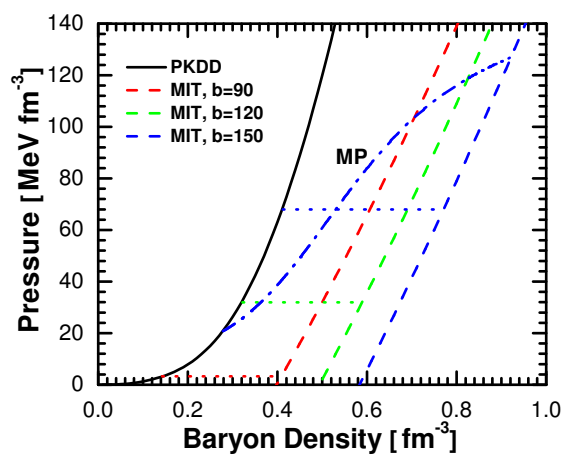

(a)

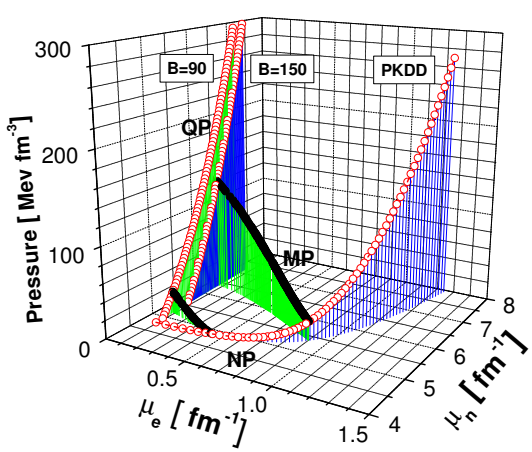

(b)

Fig. 3. (a) Pressure vs. baryon density of $\beta$-stable neutron star matter for different EoSs. The dotted lines show the hadron-quark phase transition in Maxwell construction for different bag constants, while the dot-dashed line show the result in Gibbs construction for $B=150 \mathrm{MeV} / \mathrm{fm}^{3}$. (b) Gibbs phase construction of a two-component system. The red circle NP and QP show the pressure of the hadronic and the quark phase under the condition of charge neutrality. The solid black curves MP correspond to the mixed phase for different bag constants. 


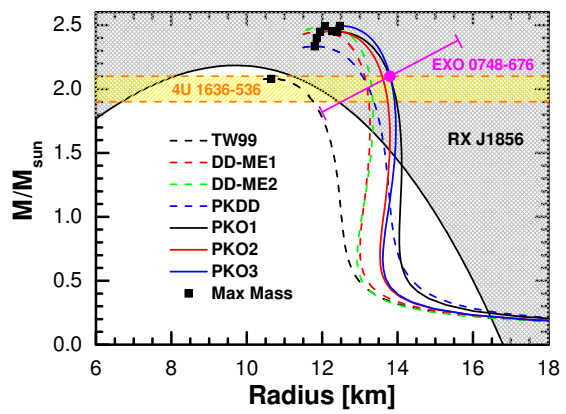

(a)

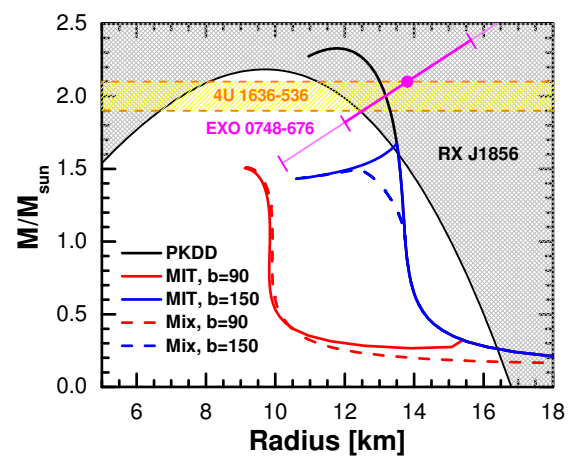

(b)

Fig. 4. Mass-radius relations for compact stars corresponding to (a) different nuclear matter EoSs. (b) different hybrid star EoSs (DDRMF+MIT). The observational constraints are taken from Ref. 1,2 and therein.

will emerge where both hadron phase and quark phase coexist. From Fig. 3 (a) we can see the plateau for pressure has been destroyed when the Gibbs construction is considered. In the mixed phase, the pressure is the same in the two phases to ensure mechanical stability, and goes up continuously with increasing baryon density. In Fig. 33(b) are shown the pressure routes in two chemical potential component plane. While the neutron density goes up, the electron chemical potential increases merely in pure hadron phase. The emergence of quark matter makes it to decrease in mixed phase and approach to zero in pure quark phase. During the total process, the pressure always raises monotonously up. Moreover, from the figure one can see, given the lager bag constants, the transition to occur at higher baryon density.

Fig. 4 (b) are shown the mass-radius relations using different hybrid star EoSs. Both two constructions for the phase transition are used. It has been found that different constructions influence very little the final mass-radius relation of massive neutron stars. Within the MIT bag model with constant $B$, the maximum mass of a neutron star never exceeds a value of about $1.6 \mathrm{M}_{\odot}$, and the star radius will increase when $B$ goes up. These results are not in agreement with recent observational constraints. Therefore, more refined quark models are necessary in further studies.

\section{Conclusions}

In conclusion, we studied neutron star properties based on the DDRHF theory, and compared with recent observational data of neutron stars. For maximum masses of neutron stars, theoretical results $2.3 \mathrm{M}_{\odot} \leq \mathrm{M}_{\max } \leq 2.5 \mathrm{M}_{\odot}$ could reproduce recent observational constraint. However, DDRHF gives larger proton fractions at high baryon density for neutron star matter than ones from DDRMF, which are not consistent with D-Urca constraint. Then the hadron-quark phase transition is 
studied by using the MIT bag model. Different transition constructions give similar maximum masses. While bag constant decreases, the maximum mass will increase but can never be larger than $1.6 \mathrm{M}_{\odot}$, meanwhile the radius of stars will be reduced.

\section{Acknowledgments}

We thank H.-J. Schulze for the stimulating discussions at Catania University. This work is partly supported by Asia-Europe Link Program in Nuclear Physics and Astrophysics (CN/ASIA-LINK/008 094-791), Major State Basic Research Developing Program 2007CB815000 as well as the National Natural Science Foundation of China under Grant No. 10435010, 10775004 and 10221003.

\section{References}

1. T. Klähn, D. Blaschke, S. Typel et al., Phys. Rev. C 74, 035802 (2006).

2. T. Klähn, D. Blaschke, F. Sandin et al., arXiv:nucl-th/0609067v2.

3. B. D. Serot, J. D. Walecka Adv. Nucl. Phys. 16, 1 (1986).

4. P. G. Reinhard Rep. Prog. Phys. 52, 439 (1989).

5. P. Ring, Prog. Part. Nucl. Phys. 37, 193 (1996).

6. S. Typel, H. H. Wolter, Nucl. Phys. A 656, 331-364 (1999).

7. T. Nikšić, D. Vretenar, P. Finelli et al., Phys. Rev. C 66, 024306 (2002).

8. G. A. Lalazissis, T. Nikšić, D. Vretenar et al., Phys. Rev. C 71, 024312 (2005).

9. W. H. Long, J. Meng, N. V. Giai et al., Phys. Rev. C 69, 034319 (2004).

10. A. Bouyssy, S. Marcos, J. F. Mathiot et al., Phys. Rev. Lett. 55, 1731 (1985).

11. A. Bouyssy, J. F. Mathiot, N. Van Giai, and S. Marcos, Phys. Rev. C 36, 380 (1987).

12. P. Bernardos, V. N. Fomenko, N. V. Giai et al., Phys. Rev. C 48, 2665 (1993).

13. S. Marcos, L. N. Savushkin, V. N. Fomenko et al., J. Phys. G 30, 703 (2004).

14. W. H. Long, N. V. Giai, and J. Meng, Phys. Lett. B 640, 150 (2006).

15. W. H. Long, N. Van Giai, and J. Meng, in preparation; W. H. Long, Ph.D. thesis, Université Paris-Sud (2005, unpublished).

16. W. H. Long, N. Van Giai, and J. Meng, arXiv:nucl-th/0608009.

17. J.M. Lattimer and M. Prakash, Astrophys. J. 550, 426 (2001).

18. F. Weber, Prog. Part. Nucl. Phys. 54, 193 (2005).

19. M. Buballa, Phys. Rep. 407, 205 (2005).

20. P. Danielewicz, R. Lacey, and W. G. Lynch, Science 298, 1592 (2002).

21. D.J. Nice, E.M. Splaver, I.H. Stairs et al., Astrophys. J. 634, 1242 (2005).

22. J. E. Trümper, V. Burwitz, F. Haberl et al., Nucl. Phys. Proc. Suppl. 132, 560 (2004).

23. D. Barret, J. F. Olive and M. C. Miller, Mon. Not. Roy. Astron. Soc. 361, 855 (2005).

24. F. Özel, Nature 441, 1115 (2006)

25. A. Chodos, R. L. Jaffe, K. Johnson et al., Phys. Rev. D 9, 3471 (1974).

26. E. Witten, Phys. Rev. D 30, 272 (1984); G. Baym, E. W. Kolb, L. McLerran, T. P. Walker, and R. L. Jaffe, Phys. Lett. B 160, 181 (1985); N. K. Glendenning, Mod. Phys. Lett. A 5, 2197 (1990).

27. E. Fahri and R. L. Jaffe, Phys. Rev. D 30, 2379 (1984).

28. G. F. Burgio, M. Baldo, P. K. Sahu et al., Phys. Lett. B 526, 19 (2002); G. F. Burgio, M. Baldo, P. K. Sahu, and H.-J. Schulze, Phys. Rev. C 66, 025802 (2002).

29. D. Blaschke, H. Grigorian, and D. Voskresensky, Astron. Astrophys. 424, 979 (2004).

30. D. Blaschke, H. Grigorian, Prog. Part. Nucl. Phys. 59, 139 (2007).

31. N. K. Glendenning, Phys. Rev. D 46, 1274 (1992). 